\title{
Downlink Beamforming for DS-CDMA Mobile Radio with Multimedia Services
}

\author{
Ying-Chang Liang, Senior Member, IEEE, Francois P. S. Chin, and K. J. Ray Liu
}

\begin{abstract}
Downlink beamforming is a promising technique for direct-sequence code-division multiple-access (DS-CDMA) systems with multimedia services to effectively reduce strong interferences induced by high data rate users. In this paper, a new downlink beamforming technique is proposed that converts downlink beamforming problem into a virtual uplink one and takes into account of the data rate information of all users. Since the main complexity of this method is due to the existence of multidelay paths, two simplified algorithms are suggested using equivalent one-path channel vector to replace multipath channel vectors. Computer simulation results are given to evaluate downlink capacity of DS-CDMA systems using base station antenna array and the new algorithms proposed in this paper.
\end{abstract}

Index Terms-Adaptive beamforming, code-division multiple access, multimedia communications, power control.

\section{INTRODUCTION}

W IDE-BAND direct-sequence code-division multiple-access (DS-CDMA) is a promising radio access technique for the third-generation mobile communication systems due to its flexibility to support a variety of voice, video, and data services. These services will require higher data rates and higher received signal power levels, thus creating larger interference between users. The interference levels have to be reduced effectively in order to obtain high system capacity. Spatial-division multiple access (SDMA) [1]-[3], by which a plurality of antenna elements are equipped at the base station in order to receive and transmit data information from and to the desired user, has been proposed as an effective technique to suppress interferences. The main operations in SDMA include uplink (from mobile station to base station) beamforming and downlink (from base station to mobile station) beamforming. Uplink beamforming consists of uplink beamforming weight generation, and uplink signal demultiplexing; downlink beamforming includes downlink beamforming weight generation and downlink signal multiplexing. Theoretically, in both links, the associated link channel responses are of critical importance in order to generate the relevant beamforming weights.

Uplink beamforming is easier for implementation than its downlink counterpart since the antenna array is usually

Paper approved by Z. Kostic, the Editor for Wireless Communication of the IEEE Communications Society. Manuscript received January 15, 2000; revised July 25,2000 . This paper was presented in part at the IEEE Vehicular Technology Conference, Amsterdam, The Netherlands, September 1999.

Y.-C. Liang and F. P. S. Chin are with the Centre for Wireless Communications, 117674 Singapore.

K. J. R. Liu is with the Department of Electrical Engineering and Institute for Systems Research, University of Maryland, College Park, MD 20742 USA.

Publisher Item Identifier S 0090-6778(01)05772-5. equipped at the base station. Joint beamforming and power control can further increase uplink capacity of DS-CDMA systems. In [4], power-based power control scheme and maximal-ratio combining (MRC) beamforming criterion are developed; while in [5], signal-to-interference ratio (SIR)-based power control and minimum mean-square-error (MMSE) beamforming criterion are proposed. Both methods can dramatically improve the whole system capacity as compared with single antenna case. In practice, it is also desirable to increase downlink capacity in order to improve the whole system capacity. In particular, downlink performance is even more important for the next-generation communication systems in which wireless internet, video-on-demand, and multimedia services are to be required.

One issue that complicates downlink beamforming problem is the possible lack of direct measurement of downlink channel responses at the base station, especially for frequency-divisionduplex (FDD) systems. One conceptually simple method for downlink channel estimation is probing-feedback approach [6], even though it is only applicable in environments that vary very slowly in time, and may require complete redesign of uplink and downlink protocols. Another statistical method is based on the use of direction-of-arrival (DOA) information embedded in received uplink signals [7], [8]. In fact, since uplink and downlink signals travel through reflections and deflections due to the same scatters surrounding the mobile and the base station, the DOAs of the uplink signals might be the only constant parameters which can be used for downlink channel estimation. DOA-based approaches employ the received uplink signals to compute the desired user's DOAs first; then downlink channel responses are constructed using known relations between uplink and downlink steering vectors.

The other issue complicating downlink beamforming problem is the lack of efficient downlink beamforming algorithms even though the downlink channel responses are available. In particular, it is often believed that obtaining the optimal downlink beamforming weights involves complicated multivariable optimization problem. One simple method is to set the downlink channel responses as the downlink beamforming weights. This approach, also called the MRC method, is equivalent to keeping the main beam of the downlink beam pattern toward the desired user. When uplink employs MMSE beamforming, MRC-based downlink beamforming may not be able to provide enough system capacity to match its uplink counterpart, especially for the cases in which lower data rate users are spatially closed to higher data rate users.

Recently, Rashid-Farroki et al. proposed a virtual uplink beamforming and power control technique (V-UBPCT) to 
generate downlink beamforming weights for SDMA [10], which just involves simple computations as in real uplink beamforming. The success of V-UBPCT lies in the combination of downlink beamforming and power control. From this point of view, the multivariable optimization algorithm in [20] is not a self-completed algorithm. However, the V-UBPCT algorithm of [10] cannot be applied to multimedia DS-CDMA systems directly due to the following reasons.

- In multimedia DS-CDMA systems, different users may require different quality of service (QoS), such as data rates. This information should be taken into consideration when designing the optimal downlink beamforming weights.

- In DS-CDMA systems, the existence of interfinger interference (IFI) makes downlink beamforming problem more complicated. In particular, when the downlink problem is converted into a virtual uplink one, due to the existence of IFI, the generated virtual uplink problem does not correspond to a pure uplink situation, thus is still unsolvable.

Therefore, the objective of this paper is to develop a computationally efficient downlink beamforming scheme for DS-CDMA systems with multimedia services. By making some approximations on the IFIs, and taking into account the downlink data rate information, a new V-UBPCT method is proposed to generate the downlink beamforming weights for multimedia DS-CDMA systems. This method is based on the criterion of minimizing the total transmitted power while maintaining the SIR requirements. This optimization criterion is physically meaningful from the viewpoint of downlink power limitation, as well as minimizing the interference pollution to the outer cells, thus increasing the multicell capacity. Therefore, the new V-UBPCT method yields the optimal solution for downlink beamforming in terms of power consumption as well as capacity enhancement. Moreover, based on the new V-UBPCT method, two simplified algorithms are also derived by using equivalent one-path channel vectors (EOCVs) to replace multidelay channel vectors.

This paper is organized as follows. Section II describes downlink signal spreading schemes, beamforming schemes, and the mathematical models of uplink and downlink channel responses. In Section III, the signal-to-interference plus noise ratio (SINR) expression with downlink beamforming is derived and the downlink beamforming problem is formulated. The new V-UBPCT method is proposed in Section IV, followed by two simplified downlink beamforming algorithms in Section V. In Section VI, computer simulations are presented to evaluate and compare the capacity of systems using different downlink beamforming algorithms. Finally, conclusions are drawn in Section VII.

\section{SYSTEM DESCRIPTION}

Suppose $N$ mobile users share the same sector in which an $M$-element uniform linear array (ULA) is equipped. Narrow-band signals are first spread to wide-band signals using different spreading codes, then transmitted to the desired user. A two-layered code structure that consists of short spreading codes and long scrambling codes is employed. All users sharing the same cell employ the same scrambling codes for downlink. Multicode spreading scheme is used for multirate transmission. Specifically, suppose user $k$ is with normalized data rate $r_{d}(k)$, which is the ratio of the $k$ th user's downlink data rate to the basic data rate. Let $\tilde{d}_{d, k}^{(j)}(t)$ and $\widetilde{c}_{d, k}^{(j)}(t)$ be the data signal and spreading signal of the $k$ th user's $j$ th code channel, respectively, and $P_{d, k}$ the average transmitted signal power for one code channel of user $k$. The $k$ th signal to be transmitted to mobile user $k$ is given by

$$
s_{d, k}(t)=\sqrt{P_{d, k}} \sum_{j=1}^{r_{d}(k)} \tilde{d}_{d, k}^{(j)}(t) \tilde{c}_{d, k}^{(j)}(t)
$$

for $k=1, \ldots, N$, with

$$
\begin{aligned}
& \tilde{d}_{d, k}^{(j)}(t)=\sum_{m=-\infty}^{\infty} d_{d, k}^{(j)}(m) u\left(\frac{t}{T}-m\right) \\
& \widetilde{c}_{d, k}^{(j)}(t)=\sum_{m=-\infty}^{\infty} \sum_{l=0}^{G-1} f(l+m G) c_{d, k}^{(j)}(l) u\left(\frac{t}{T_{c}}-l-m G\right)
\end{aligned}
$$

for $j=1, \ldots, r_{d}(k)$, where $f(m), m=\ldots,-1,0,1, \ldots$, represents the complex, scrambling sequences common to all users; $d_{d, k}^{(j)}(m), m=\ldots,-1,0,1, \ldots$, and $c_{d, k}^{(j)}(m), m=$ $0, \ldots, G-1$ denote the data sequence and the $G$-chip, complex short spreading sequence of the $j$ th code channel of user $k$, respectively. We also assume that $|f(l+m G)|=\left|c_{d, k}^{(j)}(l)\right|=1$, and $r_{d}(k)$ is a positive integer.

For DS-CDMA systems, there are two beamforming schemes: per-path-per-weight (PPPW) scheme [11] and per-user-per-weight (PUPW) scheme [15]. PPPW beamformer provides different weight vectors for different paths, thus both multiple-access interference (MAI) and IFI can be suppressed; however, this approach is sensitive to the path-changing problem, i.e., new multipath components may appear and old ones disappear frequently, particularly for users in motion [16]. On the other hand, PUPW scheme provides the same set of weight vector to all paths of the same user, thus, it is easy for implementation and less sensitive to the path-changing problem as compared to PPPW beamformer. For the PUPW scheme, it is usually required that the beamforming responses in all paths' directions should be in relatively high values in order to acquire sufficient signal energy, thus PUPW scheme cannot suppress the IFI efficiently. However, there do exist some cases, for instance, macrocell mobile systems, in which the performance loss due to IFI is quite close for both PPPW and PUPW schemes. This is because that in those cases the angular separation between each path of the same user is relatively small [2], thus even PPPW with perfect path search cannot efficiently suppress the IFI either. Therefore, PUPW beamforming scheme will be considered in this paper.

The mobile channel is well modeled as a wide-sense stationary uncorrelated scattering (WSSUS) channel [17]. Let $\mathbf{w}_{d, j}$ denote the downlink beamforming weight vector for user $j$, and assume mobile users have the same number of uplink 
and downlink delay paths. The received signal at mobile user $k$ is given by

$$
r_{k}(t)=\sum_{j=1}^{N} \sum_{l=1}^{L_{k}} \mathbf{w}_{d, j}^{H} \mathbf{h}_{d, k}^{(l)}(t) s_{d, j}\left(t-\tau_{d, k}^{(l)}\right)+u_{k}(t)
$$

where $\mathbf{h}_{d, k}^{(l)}(t)$ and $\tau_{d, k}^{(l)}$ represent the downlink channel vector and time delay corresponding to the $l$ th path of user $k$, respectively; $L_{k}$ is the number of delay paths of user, $k$; and $u_{k}(t)$ is additive white Gaussian noise received at mobile $k$. We assume the one-sided spectrum density of $u_{k}(t)$ is $N_{d, k}$.

To establish the channel models, we consider user $k$ 's $l$ th delay path. Let $\theta_{1}<\theta_{2}<\cdots<\theta_{p}$ be DOAs of the multipath components; let $\mathbf{a}_{u}\left(\theta_{i}\right)$ be uplink array response vector for signals arriving from the $i$ th DOA, which is given by

$$
\mathbf{a}_{u}\left(\theta_{i}\right)=\left[1, e^{\jmath 2 \pi z \sin \left(\theta_{i}\right) / \lambda_{u}}, \ldots, e^{\jmath 2(m-1) \pi z \sin \left(\theta_{i}\right) / \lambda_{u}}\right]^{T}
$$

where $z$ is antenna spacing and $\lambda_{u}$ is uplink wavelength. By denoting $\alpha_{u, k}(t)$ as the complex path strength of the signal coming from the $k$ th DOA, it is seen that the uplink channel response is given by

$$
\mathbf{h}_{u, k}^{(l)}(t)=\sum_{i=1}^{p} \alpha_{u, i}(t) \mathbf{a}_{u}\left(\theta_{i}\right) .
$$

Note $\alpha_{u, i}(t)=K_{u, k} \hat{\alpha}_{u, i}(t)$, where $K_{u, k}$ is the combined uplink shadowing and path loss parameter for user $k$, and $\hat{\alpha}_{u, i}(t)$ accounts for uplink fast fading effect.

For FDD systems, according to reciprocal law, only the DOAs remain unchanged for uplink and downlink transmissions [2], [8], [18]. Thus, the downlink channel response can be written as

$$
\mathbf{h}_{d, k}^{(l)}(t)=\sum_{i=1}^{p} \alpha_{d, i}(t) \mathbf{a}_{d}\left(\theta_{i}\right)
$$

where $\mathbf{a}_{d}\left(\theta_{i}\right)$ is the downlink array response vector at DOA $\theta_{i}$, which is given by

$$
\mathbf{a}_{d}\left(\theta_{i}\right)=\left[1, e^{\jmath 2 \pi z \sin \left(\theta_{i}\right) / \lambda_{d}}, \ldots, e^{2(m-1) \pi z \sin \left(\theta_{i}\right) / \lambda_{d}}\right]^{T}
$$

with $\lambda_{d}$ being downlink wavelength, and $\alpha_{d, i}(t)=$ $K_{d, k} \hat{\alpha}_{d, i}(t)$ is the complex path strength of the signal leaving for the $k$ th DOA with $K_{d, k}$ being the combined downlink shadowing and path loss parameter for user $k$, and $\hat{\alpha}_{d, i}(t)$ accounts for downlink fast fading effect.

\section{DOWNLINK BEAMFORMING}

In uplink, both beamformer and RAKE receiver are implemented in base station [4], [11], [19]. In downlink, however, beamformer and RAKE receiver are equipped in base station and mobile terminal, respectively. Nevertheless, we still need to use the detection quality at the mobile receiver to assess the performance of the downlink beamformer implemented at the base station.

\section{A. SIR Formula at the Mobile Terminal}

Assume that the $k$ th mobile receiver knows the exact time delays, $\tau_{d, k}^{(l)}$, s, of the multipath signals. The despread output of the $l$ th path of the $k$ th user's $p$ th code channel is given by

$$
z_{d, k}^{(p, l)}(n)=\frac{1}{T_{c}} \int_{n T+\tau_{d, k}^{(l)}}^{(n+1) T+\tau_{d, k}^{(l)}} r_{k}(t) \tilde{c}_{d, k}^{(p) *}\left(t-\tau_{d, k}^{(l)}\right) d t .
$$

We consider $L_{k}$-finger RAKE combiner using MRC, and denote the RAKE coefficients as

$$
\alpha_{(j, k)}^{(i)}=\mathbf{w}_{d, j}^{H} \mathbf{h}_{d, k}^{(i)}
$$

for $i=1, \ldots, L_{k}$. The RAKE combiner output is

$$
z_{d, k}^{(p)}(n)=\sum_{l=1}^{L_{k}} \alpha_{(k, k)}^{(l)}{ }^{*} z_{d, k}^{(p, l)}(n) .
$$

At the combiner output, the interference plus background noise power is given by

$$
\begin{aligned}
I_{d, k}^{(p)}= & G \sum_{j=1, j \neq k}^{N} P_{d, j} r_{d}(j) \\
& \cdot\left(\sum_{l=1}^{L_{k}}\left|\alpha_{(k, k)}^{(l)}\right|^{2} \sum_{i=1}^{L_{k}}\left|\alpha_{(j, k)}^{(i)}\right|^{2}\right. \\
& \left.\quad-\sum_{l=1}^{L_{k}}\left|\alpha_{(k, k)}^{(l)}\right|^{2}\left|\alpha_{(j, k)}^{(l)}\right|^{2}\right) \\
& +G P_{d, k} r_{d}(k) \sum_{l=1}^{L_{k}} \sum_{i=1, i \neq l}^{L_{k}}\left|\alpha_{(k, k)}^{(l)}\right|^{2}\left|\alpha_{(k, k)}^{(i)}\right|^{2} \\
& +G^{2} \frac{N_{d, k}}{T} \sum_{l=1}^{L_{k}}\left|\alpha_{(k, k)}^{(l)}\right|^{2}
\end{aligned}
$$

where the first and second terms of the above equation correspond to IFI and MAI, respectively, the last term is due to background noise. The instantaneous signal power of RAKE combiner output is

$$
S_{d, k}^{(p)}=G^{2} P_{d, k}\left(\sum_{l=1}^{L_{k}}\left|\alpha_{(k, k)}^{(l)}\right|^{2}\right)^{2} .
$$

Note $I_{d, k}^{(p)}$ and $S_{d, k}^{(p)}$ are independent of the code channel number $p$, thus we omit $p$ from them, and each code channel of user $k$ has same instantaneous SIR, which is given by

$$
\operatorname{SIR}_{d, k}=S_{d, k} / I_{d, k} .
$$

Although the above SIR formula is derived by assuming that the normalized data rates are positive integer, it is still satisfied when they are noninteger positive number. This is consistent with the effect of voice activity. The simulation results given in Section VI are based upon noninteger normalized data rates.

\section{B. SIR-Based Power Control}

In practice, SIR-based power control is required for CDMA systems. If all users require same bit-error rate quality, we try 
to maintain $\operatorname{SIR}_{d, k}=\gamma_{d, 0}$ for each $k$, where $\gamma_{d, 0}$ is the target SIR threshold. Inserting (9), (11), and (12) into (13), and setting $\mathrm{SIR}_{d, k}=\gamma_{d, 0}$ for each $k$, we have

$$
\left(\mathbf{I}-\gamma_{d, 0} \mathbf{F}_{d}\right) \mathbf{p}_{d}=\gamma_{d, 0} \mathbf{g}_{d}
$$

where

$$
\begin{aligned}
\mathbf{F}_{d} & =\mathrm{DFR} \\
\mathbf{p}_{d} & =\left[\frac{P_{d, 1} T}{N_{0}}, \ldots, \frac{P_{d, N} T}{N_{0}}\right]^{T}
\end{aligned}
$$

and

$$
\mathbf{g}_{d}=\left[\frac{N_{d, 1} / N_{0}}{\sum_{l=1}^{L_{1}}\left|\mathbf{w}_{d, 1}^{H} \mathbf{h}_{d, 1}^{(l)}\right|^{2}}, \ldots, \frac{N_{d, N} / N_{0}}{\sum_{l=1}^{L_{N}}\left|\mathbf{w}_{d, N}^{H} \mathbf{h}_{d, N}^{(l)}\right|^{2}}\right]^{T}
$$

with

$$
\begin{aligned}
& \mathbf{D}=\operatorname{diag}\left[\frac{1}{\sum_{l=1}^{L_{1}}\left|\mathbf{w}_{d, 1}^{H} \mathbf{h}_{d, 1}^{(l)}\right|^{2}}, \ldots, \frac{1}{\sum_{l=1}^{L_{N}}\left|\mathbf{w}_{d, N}^{H} \mathbf{h}_{d, N}^{(l)}\right|^{2}}\right] \\
& \mathbf{R}=\operatorname{diag}\left[r_{d}(1), \ldots, r_{d}(N)\right]
\end{aligned}
$$

and

$$
\left\{\begin{array}{c}
\frac{1}{G}\left(\sum_{l=1}^{L_{i}}\left|\mathbf{w}_{d, i}^{H} \mathbf{h}_{d, i}^{(l)}\right|^{2}-\frac{\sum_{l=1}^{L_{i}}\left|\mathbf{w}_{d, i}^{H} \mathbf{h}_{d, i}^{(l)}\right|^{4}}{\sum_{l=1}^{L_{i}}\left|\mathbf{w}_{d, i}^{H} \mathbf{h}_{d, i}^{(l)}\right|^{2}}\right) \\
\text { if } i=j \\
\frac{1}{G}\left(\begin{array}{c}
\sum_{l=1}^{L_{i}}\left|\mathbf{w}_{d, j}^{H} \mathbf{h}_{d, i}^{(l)}\right|^{2}-\frac{\sum_{l=1}^{L_{i}}\left|\mathbf{w}_{d, i}^{H} \mathbf{h}_{d, i}^{(l)}\right|^{2}\left|\mathbf{w}_{d, j}^{H} \mathbf{h}_{d, i}^{(l)}\right|^{2}}{\sum_{l=1}^{L_{i}}\left|\mathbf{w}_{d, i}^{H} \mathbf{h}_{d, i}^{(l)}\right|^{2}} \\
\text { if } i \neq j .
\end{array}\right)
\end{array}\right.
$$

In (14)-(20), $\mathbf{p}_{d}$ is called downlink transmitted power vector, the $k$ th element of which is the one code channel signal-energy-per-bit to noise ratio for user $k ; \mathbf{R}$ is the downlink data rate matrix. For a given set of channel responses and downlink beamforming weights, if and only if $\gamma_{d, 0}<\left(1 / \rho\left(\mathbf{F}_{d}\right)\right)$, where $\rho\left(\mathbf{F}_{d}\right)$ is the spectral radius of $\mathbf{F}_{\boldsymbol{d}}$, there exists a positive power vector $\mathbf{p}_{d}$, where $\mathbf{p}_{d}=\gamma_{d, 0}\left(\mathbf{I}-\gamma_{d, 0} \mathbf{F}_{d}\right)^{-1} \mathbf{g}_{d}$, such that all $\mathrm{SIR}_{d, k}$ 's are equal to $\gamma_{d, 0}$.

\section{Formulation of Downlink Beamforming Problem}

When downlink channel responses and beamforming weights are known a priori, the above centralized power control can be used to adjust transmitted powers in order for all users to work at the given SIR target. If power constraint is not considered, $\left(1 / \rho\left(\mathbf{F}_{d}\right)\right)$ is actually the maximum achievable SIR threshold for given downlink beamforming weight vectors. Let $\gamma_{d, 0}$ be the target SIR threshold, one may define the outage probability as

$$
P_{\text {out }}=\operatorname{Pr}\left\{\frac{1}{\rho\left(\mathbf{F}_{d}\right)}<\gamma_{d, 0}\right\}
$$

Theoretically, the objective of downlink beamforming is, for given $\gamma_{d, 0}$, to choose a set of beamforming weights $\mathbf{w}_{d, k}$ 's, such that $P_{\text {out }}$ is minimal, or maximum number of users can be supported within the same sector. As $P_{\text {out }}$ is most probably affected by the cases whose $\left(1 / \rho\left(\mathbf{F}_{d}\right)\right)$ value is near $\gamma_{d, 0}$, the objective is equivalent to find a set of weight vectors such that $\left(1 / \rho\left(\mathbf{F}_{d}\right)\right)$ value is maximized for those cases, or generally, minimum total transmitted power $\mathbf{1}^{T} \mathbf{R} \mathbf{p}_{d}$ is required in order to achieve the SIR target.

It is also physically reasonable to find the downlink beamforming weights by minimizing the total transmitted power. First, in downlink, the power constraint is defined as the maximum transmitted power provided by the base station, thus we need to keep the total transmitted power as small as possible. Second, for multicell systems, lowering the total transmitted power can minimize the interference pollution to other cells, thereby improving the performance of the whole cellular system.

The process to obtain the above solution, which is the optimal solution, is a difficult multivariable optimization problem, and to the best of our knowledge, there are no any efficient techniques to solve this problem. In the next section, the downlink beamforming will be converted into an easily solved problem by making some approximations.

\section{V-UBPCT}

Since the optimal weight vector $\mathbf{w}_{d, i}$ generates almost equal beam responses at the DOAs of all delay paths of user $i$, we make the approximation that $\left|\mathbf{w}_{d, i}^{H} \mathbf{h}_{d, i}^{(k)}\right|^{2} \approx\left|\mathbf{w}_{d, i}^{H} \mathbf{h}_{d, i}^{(l)}\right|^{2}$, for $k \neq l$, then $[\mathbf{F}]_{i, j}$ in (20) approaches

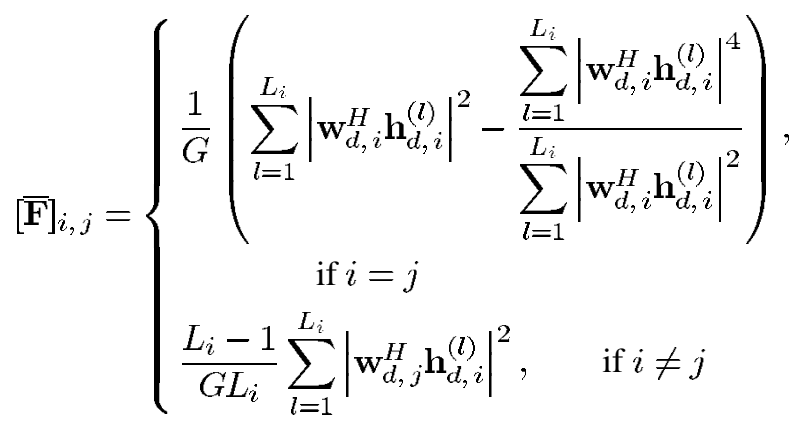

therefore, $\overline{\mathbf{F}} \approx \mathbf{F}$, and $\rho\left(\mathbf{D} \overline{\mathbf{F}}^{T} \mathbf{R}\right) \approx \rho\left(\mathbf{D F}^{T} \mathbf{R}\right)$.

Note $\rho\left(\mathbf{F}_{d}\right)=\rho(\mathbf{D F R})=\rho\left(\mathbf{D F}^{T} \mathbf{R}\right)$ since both $\mathbf{D}$ and $\mathbf{R}$ are diagonal matrices. Constructing $\overline{\mathbf{F}}_{u}=\mathbf{D} \overline{\mathbf{F}}^{T} \mathbf{R}$, we have $\rho\left(\mathbf{F}_{d}\right) \approx \rho\left(\overline{\mathbf{F}}_{u}\right)$. With this relation, the downlink beamforming problem can be solved using the idea of V-UBPCT [10]. 
Below, we first outline the algorithm steps of V-UBPCT and then provide the detailed analysis for each step; finally, we discuss the near-far problem. In order to reveal the one-to-one correspondence between real downlink and virtual uplink, we will use the same letters in virtual uplink, but put an upper bar accent $\overline{(\cdot)}$ and/or small subscript $(\cdot)_{u}$ when necessary. For example, in real downlink, we have $\mathbf{F}_{d}$; while in virtual uplink, $\overline{\mathbf{F}}_{u}$ is used.

\section{A. Algorithm Steps}

V-UBPCT consists of the following steps.

Algorithm A (V-UBPCT Algorithm):

Step A.1) Choose an initial virtual uplink power vector.

Step A.2) Compute the optimal virtual uplink weight vectors for given virtual power vector.

Step A.3) Compute the virtual power vector for given virtual weight vectors.

Step A.4) Iteratively update A.2) and A.3) until the power and weight vectors are converged. The converged weight vectors are then used as downlink beamforming weight vectors.

\section{B. Analysis of Step A.2)}

Denote $\bar{P}_{u, k}^{(n)}$ as the virtual uplink power of user $k$ at the $n$th iteration. Let $\overline{\mathbf{w}}_{u, k}$ be the $k$ th user's virtual uplink beamforming weight vector, and denote the RAKE combiner coefficients in virtual uplink as

$$
\bar{\beta}_{u, k}^{(l)}=\overline{\mathbf{w}}_{u, k}^{H} \mathbf{h}_{d, k}^{(l)} .
$$

By using the similar methodology of [19], for given virtual uplink powers, the MMSE solution of the virtual uplink beamforming weights is given by

$$
\overline{\mathbf{w}}_{u, k}^{(o)}=\overline{\mathbf{R}}_{k}^{-1} \overline{\mathbf{r}}_{k}
$$

where

$$
\overline{\mathbf{R}}_{k}=\sum_{i=1}^{L_{k}} \sum_{j=1}^{L_{k}} \bar{\beta}_{u, k}^{(i)} * \bar{\beta}_{u, k}^{(j)} \overline{\mathbf{Q}}_{k}^{(i, j)}
$$

and

$$
\overline{\mathbf{r}}_{k}=\sum_{i=1}^{L_{k}} \bar{\beta}_{u, k}^{(i)}{ }^{*} \overline{\mathbf{q}}_{k}^{(i)}
$$

with

$$
\begin{aligned}
\overline{\mathbf{Q}}_{k}^{(l, l)}= & r_{d}(k) \bar{P}_{u, k}^{(n)} G \sum_{j \neq l} \mathbf{h}_{d, k}^{(j)} \mathbf{h}_{d, k}^{(j)}{ }^{H} \\
& +G \sum_{m \neq k} \frac{L_{m}-1}{L_{m}} r_{d}(m) \bar{P}_{u, m}^{(n)} \sum_{j} \mathbf{h}_{d, m}^{(j)} \mathbf{h}_{d, m}^{(j)}{ }^{H} \\
& +\bar{P}_{u, k}^{(n)} G^{2} \mathbf{h}_{d, k}^{(l)} \mathbf{h}_{d, k}^{(l)}{ }^{H}+\frac{N_{0}}{T} G^{2} \mathbf{I} \\
\overline{\mathbf{Q}}_{k}^{(j, l)}= & \bar{P}_{u, k}^{(n)} G^{2} \mathbf{h}_{d, k}^{(j)} \mathbf{h}_{d, k}^{(l)}{ }^{H}, \quad j \neq l
\end{aligned}
$$

and

$$
\overline{\mathbf{q}}_{k}^{(i)}={\sqrt{\bar{P}_{u, k}}}^{(n)} G \mathbf{h}_{u, k}^{(i)} .
$$

Based on (23)-(28), an iterative approach can be developed for estimating the optimum virtual uplink beamforming weights for given virtual uplink power vector.
Algorithm B (Algorithm for Estimating Optimal Virtual Uplink Beamforming Weights for Given Virtual Uplink Power Vector):

Step B.1) Choose an initial virtual weight vector, say $\overline{\mathbf{w}}_{u, k}=[1,0, \ldots, 0]^{T}$.

Step B.2) Use (23) to calculate the virtual RAKE combiner coefficients.

Step B.3) Compute $\overline{\mathbf{R}}_{k}$ and $\overline{\mathbf{r}}_{k}$ from (25)-(28), thus the virtual weight vector from (24).

Step B.4) Iteratively update B.2) and B.3) until the weight vector is converged.

\section{Analysis of Step A.3)}

With the virtual uplink beamforming weight vectors, the virtual uplink SIR formula can be shown as follows [19]:

$$
\overline{\mathrm{SIR}}_{u, k}=\frac{\frac{\bar{P}_{u, k} T}{N_{0}}}{\sum_{i=1}^{N} \bar{f}_{k, i} \frac{\bar{P}_{u, i} T}{N_{0}}+\frac{\left\|\overline{\mathbf{w}}_{u, k}\right\|^{2}}{\sum_{l=1}^{L_{k}}\left|\overline{\mathbf{w}}_{u, k}^{H} \mathbf{h}_{d, k}^{(l)}\right|^{2}}}
$$

with

$$
\bar{f}_{k, i}= \begin{cases}\frac{r_{d}(k)}{G}\left(1-\frac{\sum_{l=1}^{L_{k}}\left|\overline{\mathbf{w}}_{u, k}^{H} \mathbf{h}_{d, k}^{(l)}\right|^{4}}{\left(\sum_{l=1}^{L_{k}}\left|\overline{\mathbf{w}}_{u, k}^{H} \mathbf{h}_{d, k}^{(l)}\right|^{2}\right)^{2}}\right), & \text { if } i=k \\ \frac{\left(L_{i}-1\right) r_{d}(i)}{L_{i} G} \frac{\sum_{l=1}^{L_{i}}\left|\overline{\mathbf{w}}_{u, k}^{H} \mathbf{h}_{d, i}^{(l)}\right|^{2}}{\sum_{l=1}^{L_{k}}\left|\overline{\mathbf{w}}_{u, k}^{H} \mathbf{h}_{d, k}^{(l)}\right|^{2}}, & \text { if } i \neq k\end{cases}
$$

for $k=1, \ldots, N$. Using SIR-based power control, we try to adjust the virtual uplink powers such that the SIR at the virtual RAKE combiner output is always kept at the prescribed target value for each user, while each virtual transmitter keeps the transmitted power at the minimum required level to reduce the interference to other users, or we try to keep

$$
\left(\mathbf{I}-\gamma_{u, 0} \overline{\mathbf{F}}_{u}\right) \overline{\mathbf{p}}_{u}=\gamma_{u, 0} \overline{\mathbf{g}}_{u}
$$

where $\gamma_{u, 0}$ is the target SIR threshold in virtual uplink, $\left[\overline{\mathbf{F}}_{u}\right]_{i, j}=\bar{f}_{i, j}$

$$
\overline{\mathbf{p}}_{u}=\left[\frac{\bar{P}_{u, 1} T}{N_{0}}, \ldots, \frac{\bar{P}_{u, N} T}{N_{0}}\right]^{T}
$$

and

$$
\overline{\mathbf{g}}_{u}=\left[\frac{\left\|\overline{\mathbf{w}}_{d, 1}\right\|^{2}}{\sum_{l=1}^{L_{1}}\left|\overline{\mathbf{w}}_{d, 1}^{H} \mathbf{h}_{d, 1}^{(l)}\right|^{2}}, \ldots, \frac{\left\|\overline{\mathbf{w}}_{d, N}\right\|^{2}}{\sum_{l=1}^{L_{N}}\left|\overline{\mathbf{w}}_{d, N}^{H} \mathbf{h}_{d, N}^{(l)}\right|^{2}}\right]^{T} .
$$

Given a set of virtual uplink beamforming weights, $\overline{\mathbf{w}}_{u, k}$ 's, if and only if $\gamma_{u, 0}<\left(1 / \rho\left(\overline{\mathbf{F}}_{u}\right)\right)$, there exists a positive power vector $\overline{\mathbf{p}}_{u}$, where $\overline{\mathbf{p}}_{u}=\gamma_{u, 0}\left(\mathbf{I}-\gamma_{u, 0} \overline{\mathbf{F}}_{u}\right)^{-1} \overline{\mathbf{g}}_{u}$, such that 
$\overline{\mathrm{STR}}_{u, k}$ 's are all equal to $\gamma_{u, 0}$, while each element of the virtual power vector is minimized.

For given virtual uplink weights, the virtual uplink power vector is updated as follows:

$$
\overline{\mathbf{p}}_{u}^{(n+1)}=\gamma_{u, 0} \overline{\mathbf{F}}_{u} \overline{\mathbf{p}}_{u}^{(n)}+\gamma_{u, 0} \overline{\mathbf{g}}_{u} .
$$

\section{Convergence of Algorithm A}

Algorithm A takes into account downlink data rate information in designing downlink beamforming weights. Also, the effect of orthogonality in downlink channels is well taken care of. Algorithm A can be considered as a multirate extension of the algorithm proposed in [10], thus, the convergence analysis of this algorithm can be obtained by mimicking the proof of $[5$, Theorem 1].

\section{E. Some Considerations}

1) Several differences between real UBPCT (R-UBPCT) and V-UBPCT are as follows.

- In R-UBPCT, real uplink channel responses are involved; while in V-UBPCT, downlink channel responses are considered as virtual uplink channel responses.

- The SIR and power control formulas are different for these two cases; please refer to (29)-(31) of this paper and [19, eqs. (17)-(19)].

- Power constraint should be considered in R-UBPCT in order to evaluate the uplink capacity [19]; while in V-UBPCT, power constraint is not necessary to be considered, since the virtual uplink powers in V-UBPCT are just some weighted coefficients related in the algorithm, and they do not correspond to any real powers.

2) Although there are some differences between R-UBPCT and V-UBPCT, using similar methodology of [5], we can show that the virtual uplink power vector is element-wise minimal in V-UBPCT. With this, in Appendix A, we show that the required downlink total transmitted power $\mathbf{1}^{T} \mathbf{R} \mathbf{p}_{d}$ is minimal in order to achieve the SIR target $\gamma_{d, 0}$.

4) In order to keep the algorithm stable, during the iterations of the algorithm, we choose $\gamma_{u, 0}=\gamma_{d, 0}$ for $\gamma_{d, 0}<\left(1 / \rho\left(\overline{\mathbf{F}}_{u}\right)\right)$, and $\gamma_{u, 0}=\left(0.99 / \rho\left(\overline{\mathbf{F}}_{u}\right)\right)$ for $\gamma_{d, 0} \geq\left(1 / \rho\left(\overline{\mathbf{F}}_{u}\right)\right)$, where $\gamma_{u, 0}$ is the target SIR threshold in the virtual uplink update.

6) In uplink, the beamforming weights can be updated in symbol-by-symbol basis using adaptive algorithms, such as LMS, in order to take care of instantaneous changes of the environments [11]. In downlink, however, the beamforming weights are generated at the base station from statistical point of view, thus they should be updated and applied in slot-by-slot or multislot-by-multislot basis. Real downlink power control can be implemented via fast transmit power control scheme based upon the SIR measurement at the mobile terminals [14]. The advantages of this scheme is as follows.

- In adaptive beamforming and power control scheme, if the main beam is not formed to the desired user at the beginning, the transmitted power for that user may be increased in order to achieve the SIR target. With this process repeated and repeated, the transmitted power for that user may be diverged. With V-UBPCT algorithm, the near optimal downlink beam pattern can be formed quickly, and the main beam is directed to the desired user even at the first slot. Therefore, the antenna gain is made good use of from the beginning, and the convergence of the power control algorithm is similar to that with single antenna, and of course is guaranteed.

- The downlink beamforming weights affect the SIR measurement at the mobile terminals. By adjusting the downlink beamforming weights in slot-by-slot or multislot-bymultislot basis, it is able to obtain more accurate SIR measurement at the mobile terminals, thus to help the implementation of power control algorithm.

\section{F. Near-Far Problem}

From 6), we have $\mathbf{h}_{d, k}^{(l)}(t)=K_{d, k} \hat{\mathbf{h}}_{d, k}^{(l)}(t)$ with $\hat{\mathbf{h}}_{d, k}^{(l)}(t)=$ $\sum_{i=1}^{p} \hat{\alpha}_{d, i}(t) \mathbf{a}_{d}\left(\theta_{i}\right)$. For uniform delay profile Rayleigh fadings, we assume $\hat{\alpha}_{d, i}(t), i=1, \ldots, p$, are complex random Gaussian distribution with zero mean and variance $1 / p$.

Proposition 1: For virtual uplink beamforming and power control technique, whether $\overline{\mathbf{w}}_{u, k}$ 's are normalized or not will not affect the SIR values in (29), as well as the elements of $\overline{\mathbf{F}}_{u}$ and $\overline{\mathbf{g}}_{u}$, thus those of $\overline{\mathbf{p}}_{u}$ in (31). Therefore, virtual uplink weights are blind to constant scaler.

Theorem 1: For given DOA and fading coefficients, or $\hat{\mathbf{h}}_{d, k}^{(l)}$ 's, the optimal virtual uplink beamforming weights, i.e., the optimal downlink beamforming weights are independent of the combined shadowing and path loss parameters, $K_{d, k}$ 's.

Proof: See Appendix B.

Theorem 1 provides us an excellent property of virtual uplink technique, i.e., even though the optimal transmitted power vectors are dependent on downlink path loss parameters, these parameters are not needed in order to determine the optimal downlink beamforming weights. Thus, in practice, the downlink channel responses can be normalized when they are used in V-UBPCT algorithm. This is specifically useful when downlink channel responses are estimated from uplink ones.

\section{Two SimplifiEd AlgorithMS}

In virtual uplink beamforming, it is the multidelay effect that makes it complicated to obtain virtual uplink weight vectors for given virtual uplink power vector. Specifically, iterative approach (Algorithm B) is needed to calculate optimal weight vectors for given power vector. If there is only one path, a one-step closed-form solution exists. Base on this idea, EOCVs can be used to reduce the complexity of the involved algorithm for multidelay cases.

In order to reveal the one-to-one correspondence between V-UBPCT and our new simplified algorithms, we will use the same letters in describing the new algorithms, but put a tilde accent in the corresponding letters $(\cdot)$ when necessary. For example, in V-UBPCT, we use $\overline{\mathbf{w}}_{u, k}$ to denote virtual uplink weights of user $k$; while in the new algorithms, $\tilde{\mathbf{w}}_{u, k}$ is used.

Denote $\tilde{\mathbf{h}}_{u, k}$ as the EOCV of user $k$ and $\tilde{\mathbf{w}}_{d, k}$ as the virtual uplink beamforming weight vector of user $k$. In the simplified 
algorithms, we set $\mathbf{h}_{d, k}^{(l)}=\tilde{\mathbf{h}}_{d, k}$, for $l=1, \ldots, L_{k}$. By denoting $\tilde{\beta}_{u, k}=\tilde{\mathbf{w}}_{u, k}^{H} \tilde{\mathbf{h}}_{d, k}$, from (23)-(28), the MMSE solution of the virtual uplink beamforming weights is given by

$$
\tilde{\mathbf{w}}_{u, k}^{(o)}=\tilde{\mathbf{R}}_{k}^{-1} \tilde{\mathbf{r}}_{k}
$$

where $\tilde{\mathbf{R}}_{k}=L_{k}\left|\tilde{\beta}_{u, k}\right|^{2} \tilde{\mathbf{Q}}_{k}$ and $\tilde{\mathbf{r}}_{k}=L_{k} \tilde{\beta}_{u, k}^{*} \tilde{\mathbf{q}}_{k}$ with

$$
\begin{aligned}
\tilde{\mathbf{Q}}_{k}= & G \sum_{m \neq k}\left(L_{m}-1\right) r_{d}(m) \tilde{P}_{u, m}^{(n)} \tilde{\mathbf{h}}_{d, m} \tilde{\mathbf{h}}_{d, m}^{H} \\
& +\left(L_{k} G^{2}+\left(L_{k}-1\right) G r_{d}(k)\right) \tilde{P}_{u, k}^{(n)} \tilde{\mathbf{h}}_{d, k} \tilde{\mathbf{h}}_{d, k}^{H}+\frac{N_{0}}{T} G^{2} \mathbf{I}
\end{aligned}
$$

and

$$
\tilde{\mathbf{q}}_{k}=\sqrt{\tilde{P}_{u, k}^{(n)}} G \tilde{\mathbf{h}}_{d, k} .
$$

Since whether $\tilde{\mathbf{w}}_{u, k}^{(o)}$, s are normalized or not will not affect the SIR values, the MMSE solution of the virtual uplink beamforming weights can also be computed as

$$
\tilde{\mathbf{w}}_{u, k}^{(o)}=\tilde{\mathbf{Q}}_{k}^{-1} \tilde{\mathbf{q}}_{k} .
$$

The EOCV can be estimated by choosing the principal eigenvector of the user's downlink channel covariance matrix (DCCM), which is defined

$$
\begin{aligned}
\mathbf{R}_{d, k}=\mathrm{E}[ & {\left[\mathbf{h}_{d, k}^{(1)}(t), \ldots, \mathbf{h}_{d, k}^{\left(L_{k}\right)}(t)\right] } \\
& \left.\cdot\left[\mathbf{h}_{d, k}^{(1)}(t), \ldots, \mathbf{h}_{d, k}^{\left(L_{k}\right)}(t)\right]^{H}\right]
\end{aligned}
$$

for $k=1, \ldots, N$. The DCCM is the same as the uplink channel covariance matrix (UCCM) for TDD mode; while for FDD mode, DCCM can be estimated from UCCM using frequency calibration processing. Specifically, from (5) and (6), we have

$$
\mathbf{R}_{u, k}^{(l)}=\sum_{i=1}^{p} E\left(\left|\alpha_{u, i}(t)\right|^{2}\right) \mathbf{a}_{u}\left(\theta_{k}\right) \mathbf{a}_{u}^{H}\left(\theta_{i}\right)
$$

and

$$
\mathbf{R}_{d, k}^{(l)}=\sum_{i=1}^{p} E\left(\left|\alpha_{d, i}(t)\right|^{2}\right) \mathbf{a}_{d}\left(\theta_{i}\right) \mathbf{a}_{d}^{H}\left(\theta_{i}\right) .
$$

Usually, for macrocell mobile systems, the DOAs of the multipath components of the same delay path are near one given direction. For example, in rural or suburban mobile radio, a high base station antenna array typically has a line-of-sight path to the vicinity of the mobile, with local scatters around the mobile generating signals transmitted from or received at the base station mainly within a given range of angles. Thus, we assume that all DOAs in (37) are distributed over $\left(\phi_{l}-\delta, \phi_{l}+\delta\right)$, where $\phi_{l}$ is called nominal DOA and $\delta$ the angular spread around $\phi_{l}$. Define the following two cost functions:

and

$$
J_{u, k}^{(l)}(\theta)=\mathbf{a}_{u}^{H}(\theta) \mathbf{R}_{u, k}^{(l)} \mathbf{a}_{u}(\theta)
$$

$$
J_{d, k}^{(l)}(\theta)=\mathbf{a}_{d}^{H}(\theta) \mathbf{R}_{d, k}^{(l)} \mathbf{a}_{d}(\theta) .
$$

It is easy to show that both $J_{u, k}^{(l)}(\theta)$ and $J_{d, k}^{(l)}(\theta)$ achieve maximum values at $\theta=\phi_{l}$. Also, if the angular spread $\delta$ is small, $\mathbf{a}_{u}\left(\phi_{l}\right)\left(\mathbf{a}_{d}\left(\phi_{l}\right)\right)$ approaches the principal eigenvector of $\mathbf{R}_{u, k}^{(l)}$
$\left(\mathbf{R}_{d, k}^{(l)}\right)$. Based on these observations, EOCV can be estimated through the following steps.

- Compute $\phi_{l}$ by finding maximum point of $\mathbf{a}_{u}^{H}(\theta) \mathbf{R}_{u, k}^{(l)} \mathbf{a}_{u}(\theta)$, for $l=1, \ldots, L_{k}$.

- Construct $\mathbf{a}_{d}\left(\phi_{l}\right)$, for $l=1, \ldots, L_{k}$, using (7).

- Calculate the principal eigenvector of $\hat{\mathbf{R}}_{d, k}=$ $\left[\mathbf{a}_{d}\left(\phi_{1}\right), \ldots, \mathbf{a}_{d}\left(\phi_{L_{k}}\right)\right]\left[\mathbf{a}_{d}\left(\phi_{1}\right), \ldots, \mathbf{a}_{d}\left(\phi_{L_{k}}\right)\right]^{H}$, and set it as $\tilde{\mathbf{h}}_{d, k}$.

Two simplified algorithms using EOCVs are proposed as follows: iterative virtual uplink power weighted (IVPW) algorithm and virtual uplink power weighted (VPW) algorithm.

IVPW Algorithm:

Step C.1) Calculate EOCVs, $\tilde{\mathbf{h}}_{d, k}, k=1, \ldots, N$.

Step C.2) Choose a set of initial virtual uplink weight vectors, say $\tilde{\mathbf{w}}_{u, k}=\tilde{\mathbf{h}}_{d, k}$.

Step C.3) Use (31) to compute the virtual power vector $\tilde{\mathbf{p}}_{u}$ for given weight vectors by setting $\tilde{f}_{k, i}=\left(\left(L_{k}-\right.\right.$ 1) $\left.r_{d}(k) / L_{k} G\right)$ for $k=i$

$$
\tilde{f}_{k, i}=\frac{\left(L_{i}-1\right) r_{d}(i)\left|\tilde{\mathbf{w}}_{u, k}^{H} \tilde{\mathbf{h}}_{d, i}\right|^{2}}{L_{k} G\left|\tilde{\mathbf{w}}_{u, k}^{H} \tilde{\mathbf{h}}_{d, k}\right|^{2}}
$$

for $k \neq i$ and

$$
\tilde{\mathbf{g}}_{u}=\left[\frac{\left\|\tilde{\mathbf{w}}_{u, 1}\right\|^{2}}{L_{1}\left|\tilde{\mathbf{w}}_{u, 1}^{H} \tilde{\mathbf{h}}_{d, 1}\right|^{2}}, \ldots, \frac{\left\|\tilde{\mathbf{w}}_{u, N}\right\|^{2}}{L_{N}\left|\tilde{\mathbf{w}}_{u, N}^{H} \tilde{\mathbf{h}}_{d, N}\right|^{2}}\right]^{T} .
$$

Step C.4) Use (34)-(36) to calculate the optimal weight vectors, $\tilde{\mathbf{w}}_{u, k}$ 's, for given power vector $\tilde{\mathbf{p}}_{u}$.

Step C.5) Iteratively update C.3) and C.4) until the power and weight vectors are converged. The converged weight vectors are used as downlink beamforming weight vectors.

The VPW algorithm is even simpler than the IVPW in the sense that no iteration is required. The virtual uplink power vector in VPW is derived from (31) by using MRC weights as the downlink weights, i.e., $\overline{\mathbf{w}}_{u, k}=\tilde{\mathbf{h}}_{d, k}$.

VPW Algorithm:

Step D.1) Calculate EOCVs, $\tilde{\mathbf{h}}_{d, k}, k=1, \ldots, N$.

Step D.2) Use (31) to compute the virtual power vector for given weight vectors by setting $\tilde{\mathbf{w}}_{u, k}=\widetilde{\mathbf{h}}_{d, k}$

$$
\begin{gathered}
\tilde{\mathbf{g}}_{u}=\left[\frac{\left\|\tilde{\mathbf{w}}_{u, 1}\right\|^{2}}{L_{1}\left|\tilde{\mathbf{w}}_{u, 1}^{H} \tilde{\mathbf{h}}_{d, 1}\right|^{2}}, \ldots, \frac{\left\|\tilde{\mathbf{w}}_{u, N}\right\|^{2}}{L_{N}\left|\tilde{\mathbf{w}}_{u, N}^{H} \tilde{\mathbf{h}}_{d, N}\right|^{2}}\right]^{T} \\
\text { and } \tilde{f}_{k, i}=\left(\left(L_{k}-1\right) r_{d}(k) / L_{k} G\right) \text { for } k=i \\
\tilde{f}_{k, i}=\frac{\left(L_{i}-1\right) r_{d}(i)\left|\tilde{\mathbf{w}}_{u, k}^{H} \tilde{\mathbf{h}}_{d, i}\right|^{2}}{L_{k} G\left|\tilde{\mathbf{w}}_{u, k}^{H} \tilde{\mathbf{h}}_{d, k}\right|^{2}}
\end{gathered}
$$

for $k \neq i$.

Step D.3) Use (34)-(36) to calculate the optimal weight vectors, and set them as downlink beamforming weight vectors.

\section{COMPUTER SimULATIONS}

A six-element ULA is equipped at the base station for each sector (three sectors/cell). Both TDD $\left(f_{u}=f_{d}=2.0 \mathrm{GHz}\right)$ and FDD $\left(f_{u}=1.8 \mathrm{GHz}, f_{d}=2.0 \mathrm{GHz}\right)$ duplex modes are evaluated. The antenna spacing is set to be half wavelength at 
frequency $1.8 \mathrm{GHz}$ for both cases. Macrocell systems are considered in which the angular spread for each delay path is $1^{\circ}$, and the nominal DOA for each delay path of the same user is uniformly distributed within the angular separation $\Delta$. Each user is with two delay paths and the basic processing gain is $G=16$. The SIR threshold is chosen to be $6.8 \mathrm{~dB}$ for both uplink and downlink. All users are assumed to be along with the cell boundary. The system capacity is determined by the maximum number of users with which the outage probability is less or equal to $1 \%$. The two delay paths are normalized to be with same energy which is similar to the case of practical power control as in practice, the interference power is measured over many time slots [11], thus the fading effects tend to be averaged out.

We compare our IVPW, VPW algorithms with D-MRC (uplink MRC weights for downlink), FC-MRC (uplink MRC weights with frequency calibration for downlink, FDD case), and a simple maximum transmit SINR (MTSINR) criterion, which generate downlink beamforming weights by maximizing

$$
J\left(\mathbf{w}_{d, k}\right)=\frac{\left|\mathbf{w}_{d, k}^{H} \tilde{\mathbf{h}}_{d, k}\right|^{2}}{\left|\mathbf{w}_{d, k}^{H} \tilde{\mathbf{h}}_{d, k}\right|^{2}+\frac{1}{G} \sum_{j \neq k}\left|\mathbf{w}_{d, k}^{H} \tilde{\mathbf{h}}_{d, j}\right|^{2}+N_{0}|| \mathbf{w}_{d, k}^{H} \|^{2}} .
$$

D-MRC is a modified version of the algorithm proposed in [18]. FC-MRC is an nature extension of D-MRC using frequency calibration for FDD systems.

Two types of data rate distributions are considered as follows.

- Type 1: Uplink and downlink data rates are chosen to be $r_{u}(k)=0.5$ and $r_{d}(k)=1.0$, for $k=1, \ldots, N$.

- Type 2: Uplink data rates are randomly chosen from $\{0.1,0.2, \ldots, 1.0\}$ with total data rate $\sum_{i=1}^{N} r_{u}(i)=0.5 N$, while downlink data rates are from $\{0.2,0.4, \ldots, 2.0\}$ with $\sum_{i=1}^{N} r_{d}(i)=N$.

First, let us evaluate the efficiency of using EOCV to replace the multidelay path channel vectors. Fig. 1(a) and (b) shows the downlink system capacity versus the angular separation using IVPW and Algorithm A for TDD systems with Type 1 and Type 2 data rates, respectively. It is seen that even though the performance loss due to using EOCV to replace the multidelay path channels is large for large angular separation; for small angular separation $\left(\leq 6^{\circ}\right)$, IVPW and V-UBPCT have similar capacity.

Next, we assume the angular separation is $10^{\circ}$, and compare the new simplified algorithms with other existing algorithms. Fig. 2 shows the outage probability with respect to different number of users for Type 1 data rate distribution and TDD systems. It is seen that IVPW is the best among the algorithms compared, which can support 33 users, while the number of users supported by VPW, MTSINR, and D-MRC are 24, 22, and 16, respectively.

Fig. 3 shows the results obtained for FDD systems with Type 1 data rate distribution. The number of users supported by D-MRC, FC-MRC, MTSINR, VPW, and IVPW are 17, 16, 22,24 , and 31 , respectively.

For TDD mode, D-MRC directs the main beam toward the intended user accurately. While for FDD mode, D-MRC directs the main beam toward the desired user with certain DOA shift due to FDD. It is this small DOA shift that makes D-MRC in

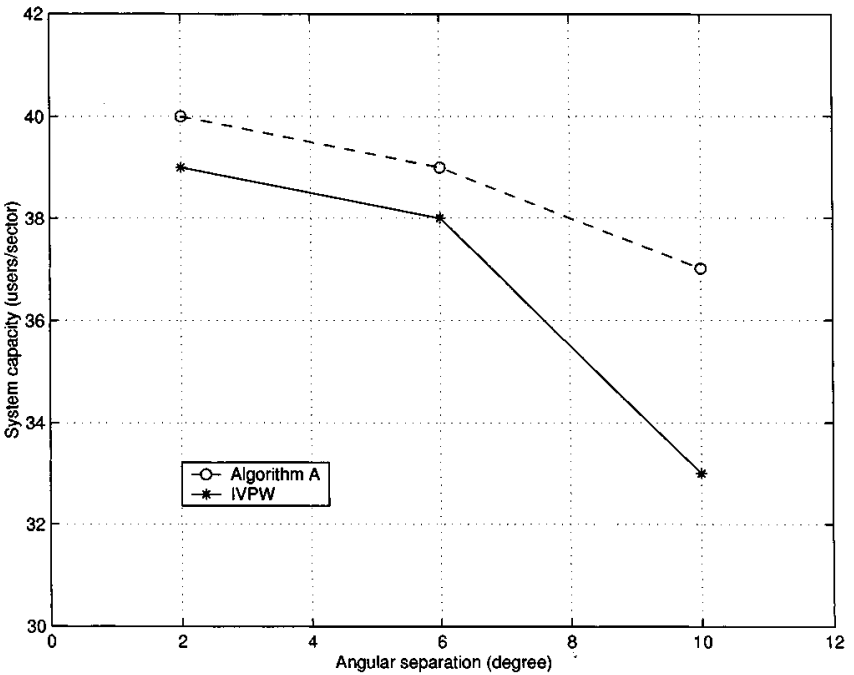

(a)

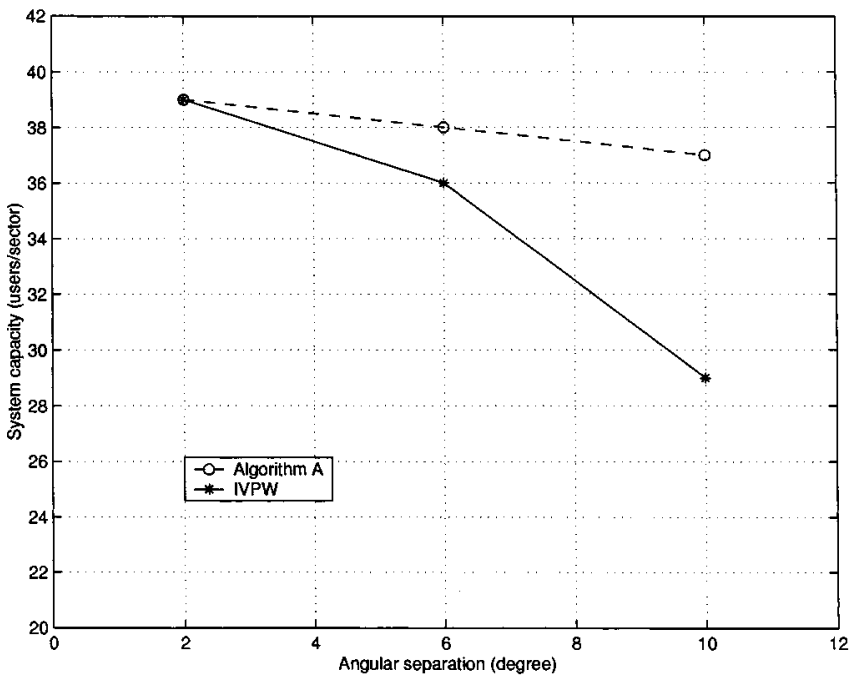

(b)

Fig. 1. System capacity versus angular separation for IVPW and Algorithm A with (a) Type 1 traffic and (b) Type 2 traffic.

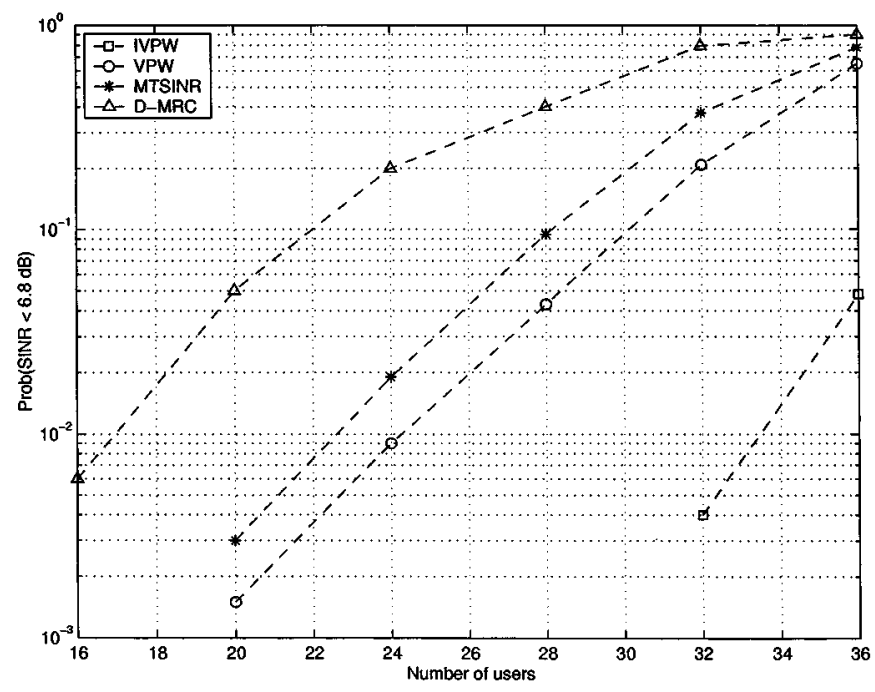

Fig. 2. Outage probability of different algirthms for Type 1 data rate distribution (TDD systems). 


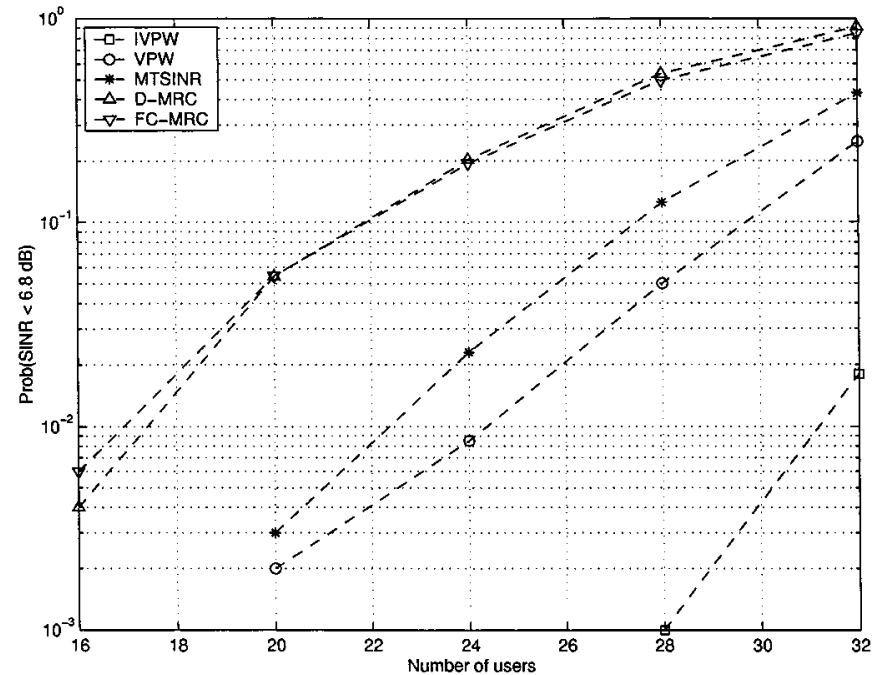

Fig. 3. Outage probability of different algirthms for Type 1 data rate distribution (FDD systems).

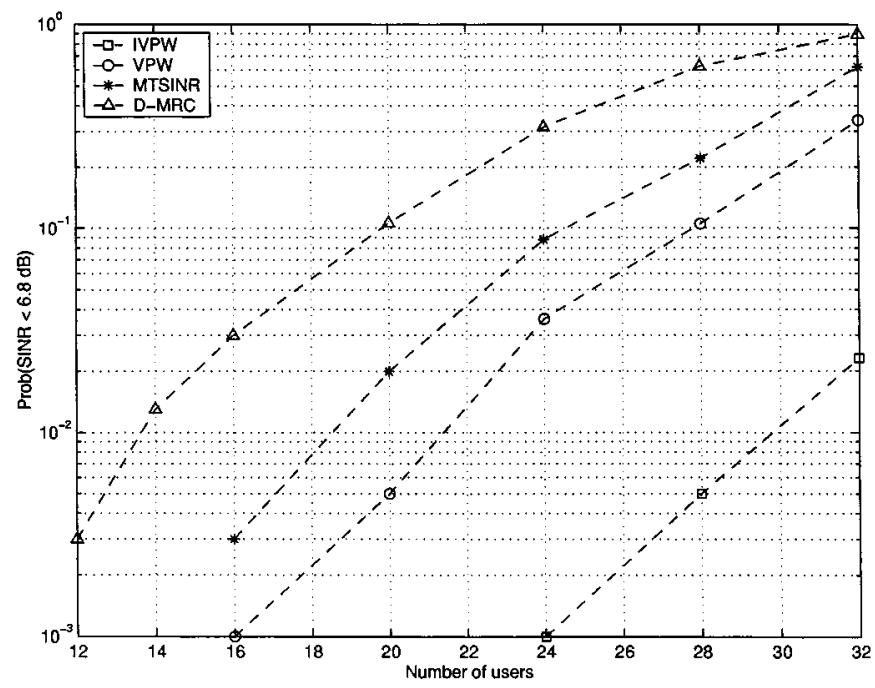

Fig. 4. Outage probability of different algirthms for Type 2 data rate distribution (TDD systems).

this environment may have certain ability to suppress interference, thus more users can be supported than those for TDD case. IVPW algorithm, which not only considers the beam responses at the intended user's directions, but also takes case of the interference polluted to the other users, provides the largest system capacity.

Figs. 4 and 5 show the outage probability with respect to different number of users (equals to the downlink total data rate) for Type 2 data rate distribution with TDD and FDD systems, respectively. The number of users supported by D-MRC, MTSINR, VPW, and IVPW are respectively $13,18,21$, and 29, for TDD systems; while for FDD systems, they are 13, 18, 20 , and 26, respectively. It is seen that using IVPW algorithm, the system capacity is more than two times of systems using $\mathrm{D}-\mathrm{MRC}$ algorithm. Also shown is that FC-MRC provides same system capacity with D-MRC for FDD systems. This is because that although FC-MRC direct its main beam toward the desired user, while D-MRC method directs its main beam toward the

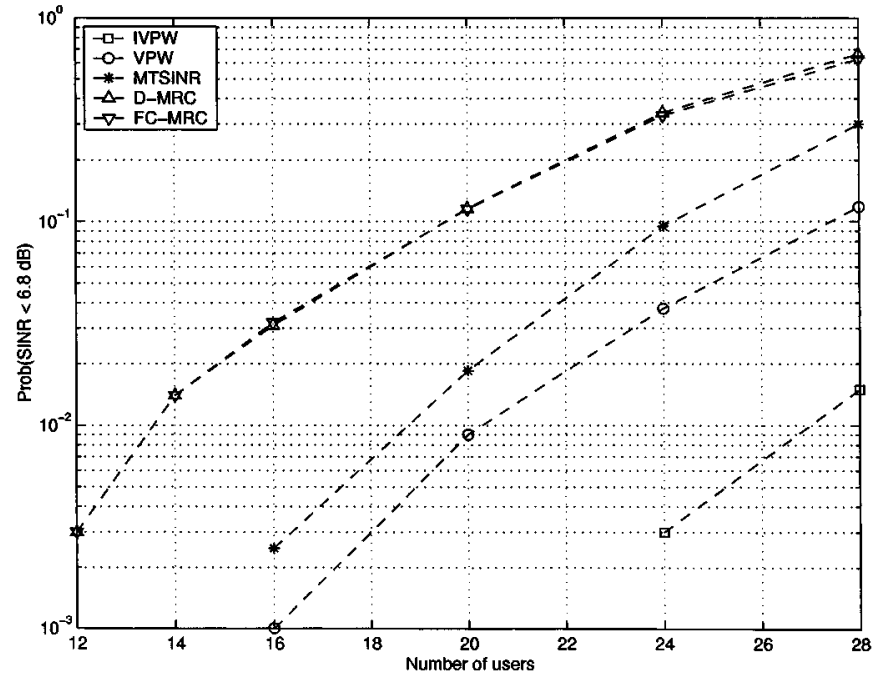

Fig. 5. Outage probability of different algirthms for Type 1 data rate distribution (FDD systems).

desired user with certain DOA shift due to FDD, this shift is not so large as the difference between uplink and downlink carrier frequency is just $10 \%$ of downlink carrier frequency.

From the simulation results, we have the following observations.

- D-MRC and FC-MRC provide smallest system capacity among the methods compared. This is because that these two methods just keep the main beam of downlink beam pattern toward the intended user (D-MRC also has certain DOA shift), but do not consider the interference polluted to other users.

- Although MTSINR method takes care of the interference pollution to other users in some extent, it fails to yield the optimal beamforming weights.

- Even though the angular separation is as high as $10^{\circ}$, the capacity enhancement of using IVPW is still obvious. In particular, for Type 1 rate distribution, the system capacity of IVPW is about 2.1 and 1.8 times that of D-MRC, for TDD and FDD, respectively; while for Type 2 rate distribution, the system capacity supported by IVPW algorithm is about 2.2 and 2.0 times of that supported by D-MRC for TDD and FDD, respectively. It is seen that for multirate services, the capacity degradation by D-MRC is very serious, while that by IVPW is slight. This is because IVPW algorithm not only employs virtual uplink concept, but also takes into account all users' downlink data rate information, thus largest system capacity can be achieved.

Finally, the average total transmitted powers required by different algorithms are shown in Tables I and II, for FDD systems with Type 1 and Type 2 data rate distributions, respectively. Here, Alg.A-T represents Algorithm A with true downlink channel responses, whose results serve as the lower bound of the average total transmitted powers. It is seen that for both cases, the required powers for both IVPW and VPW are quite near that for Alg.A-T, while D-MRC consumes largest power, and FC-MRC costs second. IVPW takes a little bit less powers than VPW at the expense of more complicated computations. 
TABLE I

Average Total Transmitted Powers for Type 1 Data Rate DISTRIBUTION USING DIFFERENT APPROACHES

\begin{tabular}{c|c|c|c|c|c|c|c|c}
\hline \hline Uвег No. & 8 & 9 & 10 & 11 & 12 & 13 & 14 & 15 \\
\hline D-MRC & $5.8256 \mathrm{~dB}$ & $6.6073 \mathrm{~dB}$ & $7.3160 \mathrm{~dB}$ & $8.0646 \mathrm{~dB}$ & $8.8289 \mathrm{~dB}$ & $9.4110 \mathrm{~dB}$ & $10.1086 \mathrm{~dB}$ & $10.8426 \mathrm{~dB}$ \\
FC-MRC & $5.3281 \mathrm{~dB}$ & $6.0842 \mathrm{~dB}$ & $6.7924 \mathrm{~dB}$ & $7.5433 \mathrm{~dB}$ & $8.1520 \mathrm{~dB}$ & $8.8096 \mathrm{~dB}$ & $9.5578 \mathrm{~dB}$ & $10.1412 \mathrm{~dB}$ \\
IVPW & $5.2635 \mathrm{~dB}$ & $5.9553 \mathrm{~dB}$ & $6.5723 \mathrm{~dB}$ & $7.2411 \mathrm{~dB}$ & $7.8456 \mathrm{~dB}$ & $8.4129 \mathrm{~dB}$ & $9.0373 \mathrm{~dB}$ & $9.5427 \mathrm{~dB}$ \\
VPW & $5.2638 \mathrm{~dB}$ & $5.9558 \mathrm{~dB}$ & $6.5733 \mathrm{~dB}$ & $7.2792 \mathrm{~dB}$ & $7.8509 \mathrm{~dB}$ & $8.4236 \mathrm{~dB}$ & $9.0458 \mathrm{~dB}$ & $9.5779 \mathrm{~dB}$ \\
Alg.A-T & $5.2345 \mathrm{~dB}$ & $5.9251 \mathrm{~dB}$ & $6.5409 \mathrm{~dB}$ & $7.2060 \mathrm{~dB}$ & $7.8041 \mathrm{~dB}$ & $8.3684 \mathrm{~dB}$ & $8.9654 \mathrm{~dB}$ & $9.4857 \mathrm{~dB}$ \\
\hline
\end{tabular}

TABLE II

Average Total Transmitted Powers For Type 2 Data Rate DistRIBUTION USING DIFFERENT APPROACHES

\begin{tabular}{c|c|c|c|c|c|c|c|c}
\hline \hline User No. & 8 & 9 & 10 & 11 & 12 & 13 & 14 & 15 \\
\hline D-MRC & $6.1752 \mathrm{~dB}$ & $6.9413 \mathrm{~dB}$ & $7.7754 \mathrm{~dB}$ & $8.7868 \mathrm{~dB}$ & $9.4693 \mathrm{~dB}$ & $10.1757 \mathrm{~dB}$ & $10.9752 \mathrm{~dB}$ & $11.8143 \mathrm{~dB}$ \\
FC-MRC & $5.6894 \mathrm{~dB}$ & $6.4330 \mathrm{~dB}$ & $7.2533 \mathrm{~dB}$ & $8.2011 \mathrm{~dB}$ & $8.9074 \mathrm{~dB}$ & $9.9223 \mathrm{~dB}$ & $10.4309 \mathrm{~dB}$ & $11.1661 \mathrm{~dB}$ \\
IVPW & $5.5305 \mathrm{~dB}$ & $6.2809 \mathrm{~dB}$ & $6.9243 \mathrm{~dB}$ & $7.6208 \mathrm{~dB}$ & $8.2300 \mathrm{~dB}$ & $8.8309 \mathrm{~dB}$ & $9.3697 \mathrm{~dB}$ & $10.0884 \mathrm{~dB}$ \\
VPW & $5.5409 \mathrm{~dB}$ & $6.2874 \mathrm{~dB}$ & $6.9399 \mathrm{~dB}$ & $7.6388 \mathrm{~dB}$ & $8.2953 \mathrm{~dB}$ & $8.8690 \mathrm{~dB}$ & $9.4301 \mathrm{~dB}$ & $10.2303 \mathrm{~dB}$ \\
Alg.A-T & $5.4994 \mathrm{~dB}$ & $6.2469 \mathrm{~dB}$ & $6.8846 \mathrm{~dB}$ & $7.5522 \mathrm{~dB}$ & $8.1648 \mathrm{~dB}$ & $8.7633 \mathrm{~dB}$ & $9.2996 \mathrm{~dB}$ & $9.9875 \mathrm{~dB}$ \\
\hline
\end{tabular}

\section{CONCLUSIONS}

Downlink beamforming is used to improve the downlink capacity of DS-CDMA systems. Virtual uplink beamforming and power control technique is developed for generating downlink beamforming weights by minimizing total transmitted power. Two simplified algorithms are suggested by using EOCV to replace multidelay path responses. Computer simulation results show that the new algorithms can provide much larger system capacity than the traditional approach that just keeps main beam toward the desired user.

\section{APPENDIX A}

In Appendix A, we will show that the total transmitted power required by the virtual uplink algorithm is minimal. Consider downlink beamforming and power control formulation shown in (14)-(20), and virtual uplink beamforming and power control formulation illustrated in (31) and its immediate sequel. Since virtual uplink weights are used as downlink beamforming weights, we have $\overline{\mathbf{w}}_{u, k}=\mathbf{w}_{d, k}$. Denote $\mathbf{1}_{w}=\left[\left\|\mathbf{w}_{1}\right\|^{2}, \ldots,\left\|\mathbf{w}_{N}\right\|^{2}\right]^{T}$, and let $\breve{\mathbf{p}}_{u}=\mathbf{R} \overline{\mathbf{p}}_{u}$, we have, from (31)

$$
\begin{aligned}
\breve{\mathbf{p}}_{u} & =\gamma_{d, 0} \mathbf{R}\left(\mathbf{I}-\gamma_{d, 0} \mathbf{D F}^{T} \mathbf{R}\right)^{-1} \mathbf{D} \mathbf{1}_{w} \\
& =\gamma_{d, 0} \mathbf{R}\left[\mathbf{D}^{-1}\left(\mathbf{I}-\gamma_{d, 0} \mathbf{D F} \mathbf{F}^{T} \mathbf{R}\right)\right]^{-1} \mathbf{1}_{w} \\
& =\gamma_{d, 0} \mathbf{R}\left(\mathbf{D}^{-1}-\gamma_{d, 0} \mathbf{F}^{T} \mathbf{R}\right)^{-1} \mathbf{1}_{w} \\
& =\gamma_{d, 0}\left(\mathbf{D}^{-1} \mathbf{R}^{-1}-\gamma_{d, 0} \mathbf{F}^{T}\right)^{-1} \mathbf{1}_{w}
\end{aligned}
$$

thus

$$
\begin{aligned}
\breve{\mathbf{p}}_{u}^{T} & =\gamma_{d, 0} \mathbf{1}_{w}^{T}\left(\mathbf{D}^{-1} \mathbf{R}^{-1}-\gamma_{d, 0} \mathbf{F}\right)^{-1} \\
& =\gamma_{d, 0} \mathbf{1}_{w}^{T} \mathbf{R}\left(\mathbf{I}-\gamma_{d, 0} \mathbf{D F R}\right)^{-1} \mathbf{D} .
\end{aligned}
$$

On the other hand, from (14), the total transmitted power is given by

$$
\mathbf{1}_{w}^{T} \mathbf{R}_{d}=\gamma_{d, 0} \mathbf{1}_{w}^{T} \mathbf{R}\left(\mathbf{I}-\gamma_{d, 0} \mathbf{D F R}\right)^{-1} \mathbf{D N}=\breve{\mathbf{p}}_{u}^{T} \mathbf{N}
$$

with $\mathbf{N}=\left[N_{d, 1} / N_{0}, \ldots, N_{d, N} / N_{0}\right]^{T}$. As the virtual uplink power vector $\breve{\mathbf{p}}_{u}$ is element-wise minimal, the total transmitted power is also minimal.
APPENDIX B PROOF OF THEOREM 1

Let

$$
\begin{aligned}
\hat{\mathbf{p}}_{u} & =\left[\frac{\bar{P}_{u, 1} T}{N_{0}} K_{u, 1}^{2}, \ldots, \frac{\bar{P}_{u, N} T}{N_{0}} K_{u, N}^{2}\right]^{T} \\
\hat{\mathbf{g}}_{u} & =\left[\frac{\left\|\overline{\mathbf{w}}_{u, 1}\right\|^{2}}{\sum_{l=1}^{L_{1}}\left|\overline{\mathbf{w}}_{u, 1}^{H} \hat{\mathbf{h}}_{d, 1}^{(l)}\right|^{2}}, \ldots, \frac{\left\|\overline{\mathbf{w}}_{u, N}\right\|^{2}}{\sum_{l=1}^{L_{N}}\left|\overline{\mathbf{w}}_{u, N}^{H} \hat{\mathbf{h}}_{d, N}^{(l)}\right|^{2}}\right]^{T}
\end{aligned}
$$

and $\left[\hat{\mathbf{F}}_{u}\right]_{i, j}=\hat{f}_{i, j}$, where $\hat{f}_{i, j} \mathrm{~s}$ are obtained via (30) with $\mathbf{h}_{d, k}^{(l)}$, s replaced by $\hat{\mathbf{h}}_{d, k}^{(l)} \mathrm{s}$. Note $\hat{\mathbf{p}}_{u}$ represents the virtual received power vector, and $\rho\left(\overline{\mathbf{F}}_{u}\right)=\rho\left(\hat{\mathbf{F}}_{u}\right)$. Then $\hat{\mathbf{p}}_{u}=\mathbf{K} \overline{\mathbf{p}}_{u}, \hat{\mathbf{g}}_{u}=\mathbf{K} \overline{\mathbf{g}}_{u}$, and $\hat{\mathbf{F}}_{u}=\mathbf{K} \overline{\mathbf{F}}_{u} \mathbf{K}^{-1}$ with $\mathbf{K}=\operatorname{diag}\left[K_{u, 1}^{2}, \ldots, K_{u, N}^{2}\right]$. Given a set of virtual uplink weights vectors, since $\hat{\mathbf{F}}_{u}$ and $\hat{\mathbf{g}}_{u}$ are independent of $K_{u, k}$ 's, the optimal virtual received power vector is also independent of $K_{u, k}$ s. Next, from (23)-(28), we have

$$
\hat{\mathbf{w}}_{u, k}^{(\circ)}=\hat{\mathbf{R}}_{k}^{-1} \hat{\mathbf{r}}_{k}
$$

where

$$
\hat{\mathbf{R}}_{k}=K_{u, k}^{2} \sum_{i=1}^{L_{k}} \sum_{j=1}^{L_{k}}\left(\hat{\beta}_{u, k}^{(i)}\right)^{*} \hat{\beta}_{u, k}^{(j)} \hat{\mathbf{Q}}_{k}^{(i, j)}
$$

and

$$
\hat{\mathbf{r}}_{k}=K_{u, k} \sum_{i=1}^{L_{k}}\left(\hat{\beta}_{u, k}^{(i)}\right)^{*} \hat{\mathbf{q}}_{k}^{(i)}
$$

with

$$
\begin{aligned}
\hat{\mathbf{Q}}_{k}^{(l, l)}= & r_{u}(k) \hat{P}_{u, k} G \sum_{j \neq l} \hat{\mathbf{h}}_{d, k}^{(j)}\left(\hat{\mathbf{h}}_{d, k}^{(j)}\right)^{H} \\
& +G \sum_{m \neq k} r_{u}(m) \hat{P}_{u, m} \sum_{j} \hat{\mathbf{h}}_{d, m}^{(j)}\left(\hat{\mathbf{h}}_{d, m}^{(j)}\right)^{H} \\
& +\hat{P}_{u, k} G^{2} \hat{\mathbf{h}}_{d, k}^{(l)}\left(\hat{\mathbf{h}}_{d, k}^{(l)}\right)^{H}+\frac{N_{0}}{T} G^{2} \mathbf{I} \\
\hat{\mathbf{Q}}_{k}^{(j, l)}= & \hat{P}_{u, k} G^{2} \hat{\mathbf{h}}_{d, k}^{(j)}\left(\hat{\mathbf{h}}_{d, k}^{(l)}\right)^{H}, \quad j \neq l
\end{aligned}
$$

and $\hat{\beta}_{u, k}^{(l)}=\hat{\mathbf{w}}_{u, k}^{H} \hat{\mathbf{h}}_{d, k}^{(l)}, \hat{\mathbf{q}}_{k}^{(i)}=\sqrt{\hat{P}_{u, k}} G \hat{\mathbf{h}}_{d, k}^{(i)}$, and $\hat{P}_{u, k}$ the $k$ th element of $\hat{\mathbf{P}}_{u}$. From Proposition 1, virtual uplink beamforming weights are blind to constant scalers, thus the optimal weight vectors are dependent on the received powers only. With these iterations carrying on, if $\gamma_{u, 0}<\left(1 / \rho\left(\hat{\mathbf{F}}_{u}\right)\right)$, there exists a set of optimal weight vectors $\left\{\hat{\mathbf{w}}_{u, 1}, \ldots, \hat{\mathbf{w}}_{u, N}\right\}$ and an optimal received power vector $\hat{\mathbf{p}}_{u}$, such that all users' SIR values are equal to $\gamma_{u, 0}$, while each received power is minimal. The optimal virtual uplink weight vectors, thus the optimal downlink weight vectors are independent of $K_{u, k} \mathrm{~s}$.

\section{REFERENCES}

[1] J. H. Winters, J. Salz, and R. D. Gitlin, "The impact of antenna diversity on the capacity of wireless communication systems," IEEE Trans. Commun., vol. 42, pp. 1740-175, Feb./Mar./Apr. 1994.

[2] A. Paulraj and C. B. Papadias, "Space-time processing for wireless communications," IEEE Signal Processing Mag., vol. 14, pp. 49-83, Nov. 1997. 
[3] S. C. Swales, M. A. Beach, D. J. Edwards, and J. P. McGeehan, "The performance enhancement of multibeam adaptive base-station antennas for cellular land mobile radio systems," IEEE Trans. Veh. Technol., vol. 39, pp. 56-67, Feb. 1990.

[4] A. F. Naguib, A. Paulraj, and T. Kailath, "Capacity improvement with base-station antenna arrays in cellular CDMA," IEEE Trans. Veh. Technol., vol. 43, pp. 691-698, Aug. 1994.

[5] F. Rashid-Farrokhi, L. Tassiulas, and K. J. R. Liu, "Joint optimal power control and beamforming in wireless networks using antenna arrays," IEEE Trans. Commun., vol. 46, pp. 1313-1324, Oct. 1998.

[6] D. Gerlach and A. Paulraj, "Adaptive transmitting antenna arrays with feedback," IEEE Signal Processing Lett., vol. 1, pp. 150-152, Oct. 1994.

[7] P. Zetterberg and B. Ottersten, "The spectrum efficiency of a base station antenna array system for spatially selective transmission," IEEE Trans. Veh. Technol., vol. 44, pp. 651-660, Aug. 1995.

[8] G. Xu and H. Liu, "An efficient transmission beamforming scheme for frequency-division-duplex digital wireless communications systems," in Proc. ICASSP'95, pp. 1729-1732.

[9] J. Salz and J. H. Winters, "Effect of fading correlations on adaptive arrays in digital wireless communications," in Proc. ICC'93, pp. $1768-1774$.

[10] F. Rashid-Farrokhi, K. J. R. Liu, and L. Tassiulas, "Transmit beamforming and power control for cellular wireless systems," IEEE J. Select. Areas Commun., vol. 16, pp. 1437-1449, Oct. 1998.

[11] S. Tanaka, M. Sawahashi, and F. Adachi, "Pilot symbol-assisted decision-directed coherent adaptive array diversity for DS-CDMA mobile radio reverse link," IEICE Trans. Fundamentals, vol. E80-A, no. 12, Dec. 1997.

[12] F. Adachi and M. Sawahashi, "Wideband multi-rate DS-CDMA for next generation mobile communication systems," in Proc. Wireless Communications Conf., Boulder, CO, Aug. 1997.

[13] F. Adachi, "Effects of orthogonal spreading and Rake combining on DS-CDMA forward link in mobile radio," IEICE Trans. Commun., vol. E80-B, no. 11, pp. 1703-1712, Nov. 1997.

[14] H. Suda, F. Kikuchi, and F. Adachi, "Effect of fast transmit power control on DS-CDMA forward link capacity," in Proc. APCC/ICCS, Singapore, 1998, pp. 413-417.

[15] Y.-C. Liang, F. Chin, and K. J. R. Liu, "Downlink beamforming for DS-CDMA mobile radio with multimedia services," in Proc. Vehicular Technology Conf. Fall'99, Amsterdam, The Netherlands, Sept. 1999, pp. $17-21$.

[16] A. J. Viterbi, CDMA: Principles of Spread Spectrum Communication. Reading, MA: Addison-Wesley, 1995.

[17] R. Steel, Mobile Radio Communications. Piscataway, NJ: IEEE Press, 1992

[18] G. G. Raleigh, S. N. Diggavi, V. K. Jones, and A. Paulraj, "A blind adaptive transmit antenna algorithm for wireless communication," in Proc. ICC'95, 1998, pp. 1494-1499.

[19] Y.-C. Liang and F. Chin, "Effect of power constraint on uplink capacity of DS-CDMA systems with antenna array and SIR-based power control," in Proc. PIMRC'99, Osaka, Japan, Sept. 1999.

[20] C. Farsakh and J. A. Nossek, "Spatial covariance based downlink beamforming in an SDMA mobile radio system," IEEE Trans. Commun., vol. 46, pp. 1497-1506, Nov. 1998.

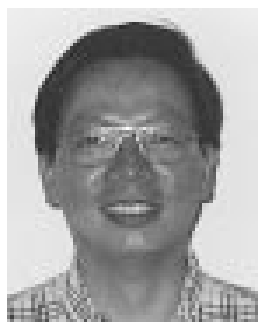

Ying-Chang Liang (SM'00) received the B.S. and Ph.D. degrees in electrical engineering from Jilin University of Technology, Changchun, China, in 1989 and 1993, respectively.

In 1997, he was a Research Associate in the Electrical Engineering Department, University of Maryland, College Park, where he conducted research on transmit diversity for IS-136 TDMA systems, closely with AT\&T Laboratories-Research. He joined the Centre for Wireless Communications (CWC), Singapore, in 1997, where he is currently a Senior Member of Technical Staff in the Communication Systems and Signal Processing Group. His research interests include adaptive signal processing for wireless communications, statistical signal processing, and higher-order statistics.

Dr. Liang received the Best Paper Award from the 50th IEEE Vehicular Technology Conference in 1999. He was also a co-recipient of the 1997 National Natural Science Award and the 1996 Science and Technology Achievement Award, both from China.

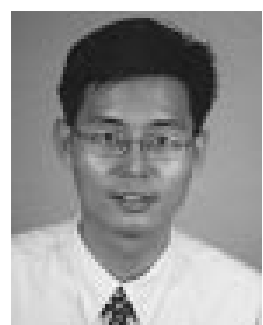

Francois P. S. Chin received the Ph.D. degree in electrical engineering from the National University of Singapore in 1996.

Since September 1995, he has been at the Centre for Wireless Communications, a government-funded research center linked to NUS, where he is now a Senior Member of Technical Staff and leads a research and development team in future broad-band wireless access. His research interests include signal processing techniques for capacity/quality enhancement and communication system design/performance

evaluation

Dr. Chin was a co-recipient of the Best Paper Award from the IEEE Vehicular Technology and Communications Conference in the Fall of 1999.

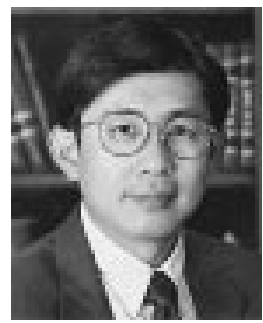

K. J. Ray Liu received the B.S. degree from the National Taiwan University in 1983, and the Ph.D. degree from the University of California at Los Angeles in 1990, both in electrical engineering.

Since 1990, he has been with the Department of Electrical and Computer Engineering and Institute for Systems Research, University of Maryland, College Park, where he is a Professor. During his sabbatical leave in 1996-1997, he was a Visiting Associate Professor at Stanford University, Stanford, CA. His research interests span broad aspects of signal processing, image/video processing, and communications in which he has published over 200 papers.

Dr. Liu received numerous awards including the 1994 National Science Foundation Young Investigator Award, the 1993 IEEE Signal Processing Society's Senior Award (Best Paper Award), the 1999 IEEE Vehicular Technology Conference Best Paper Award, the 1994 George Corcoran Award for outstanding contributions to electrical engineering education and the 1995-1996 Outstanding Systems Engineering Faculty Award in recognition of outstanding contributions in interdisciplinary research, both from the University of Maryland, and many others. He is the Editor-in-Chief of EURASIP Journal on Applied Signal Processing, and has been an Associate Editor of IEEE TRANSACTIONS ON Signal PROCESSING, a Guest Editor for the special issue on Multimedia Signal Processing of the PROCEEDING OF THE IEEE, Guest Editor for the special issue on Signal Processing for Wireless Communications of the IEEE Journal on Selected AREas in Communications, Guest Editor for the special issue on Multimedia Communications over Networks of IEEE Signal Processing Magazine, Guest Editor for the special issue on Multimedia over IP of IEEE TRANSACTIONS ON MULTIMEDIA, and an Editor of the Journal of VLSI Signal Processing Systems. He currently serves as the Chair of the Multimedia Signal Processing Technical Committee of the IEEE Signal Processing Society and the Series Editor of Marcel Dekker series on Signal Processing and Communications.. 